\title{
Consistency relation in power law G-inflation
}

\section{Sanil Unnikrishnan ${ }^{1}$ and S. Shankaranarayanan}

School of Physics, Indian Institute of Science Education and Research, Thiruvananthapuram 695016, India

E-mail: sanil@iisertvm.ac.in, shanki@iisertvm.ac.in

\begin{abstract}
In the standard inflationary scenario based on a minimally coupled scalar field, canonical or non-canonical, the subluminal propagation of speed of scalar perturbations ensures the following consistency relation: $r \leq-8 n_{T}$, where $r$ is the tensor-to-scalar-ratio and $n_{T}$ is the spectral index for tensor perturbations. However, recently, it has been demonstrated that this consistency relation could be violated in Galilean inflation models even in the absence of superluminal propagation of scalar perturbations. It is therefore interesting to investigate whether the subluminal propagation of scalar field perturbations impose any bound on the ratio $r /\left|n_{T}\right|$ in G-inflation models. In this paper, we derive the consistency relation for a class of G-inflation models that lead to power law inflation. Within these class of models, it turns out that one can have $r>-8 n_{T}$ or $r \leq-8 n_{T}$ depending on the model parameters. However, the subluminal propagation of speed of scalar field perturbations, as required by causality, restricts $r \leq-(32 / 3) n_{T}$.
\end{abstract}

Keywords: Inflation, physics of early universe, primordial gravitational waves (theory)

ArXiv ePrint: 1311.0177

\footnotetext{
${ }^{1}$ Current address: Department of Physics, The LNM Institute of Information Technology, Jaipur-302031, India. Email: sanil@lnmiit.ac.in
} 


\section{Contents}

1 Introduction 1

2 G-Inflation Preliminaries $\quad 3$

3 Power law G-Inflation 5

4 Consistency relation in Galilean Power law inflation $\quad 7$

4.1 Observational viability of Galilean power law inflation 13

5 Comparing Galilean and non-canonical scalar inflation $\quad \mathbf{1 5}$

$\begin{array}{ll}5.1 \text { In the case of power inflation } & 15\end{array}$

5.2 Generic slow roll G-inflation model 16

$\begin{array}{llr}6 & \text { Summary and conclusions } & 18\end{array}$

$\begin{array}{lr}\text { A Speed of sound of scalar field perturbations } & 20\end{array}$

\section{Introduction}

The inflationary paradigm not only heals the Big Bang theory afflicted with the horizon, flatness and monopole problems $[1,2]$, its prediction of a nearly scale invariant cosmological perturbations [3] has remarkably been verified by numerous cosmological observations with the recent one being the Cosmic Microwave Background (CMB) observation from the Planck mission $[4,5]$. In spite of this, one is yet to identify the primary source of matter field that caused inflation, although numerous viable models have been proposed [6-13]. In most of these proposed models, inflation is driven by a minimally coupled scalar field. Broadly speaking, all minimally coupled single scalar field models of inflation can be divided into the following three classes:

(i) Canonical scalar field models whose Lagrangian is of the form $\mathcal{L}=(1 / 2) \partial_{\mu} \phi \partial^{\mu} \phi-$ $V(\phi)$.

(ii) Non-canonical scalar field models [14-16] in which the Lagrangian $\mathcal{L}=\mathcal{L}(\phi, X)$ is a generic function of the field $\phi$ and the kinetic term $X=(1 / 2) \partial_{\mu} \phi \partial^{\mu} \phi$.

(iii) Galilean models of inflation or G-inflation, also known as kinetic gravity braiding models $[18,19]$, described by the Lagrangian $\mathcal{L}=K(X, \phi)+G(X, \phi) \square \phi$, where $K(X, \phi)$ and $G(X, \phi)$ can be an arbitrary function of $\phi$ and the kinetic term $X$.

In fact, the third case above is the most general class of models describing inflation and contains the other two cases. It should be noted that unlike the first two cases, the Lagrangian of the Galilean field contains the second order derivative of the field $\phi$. However, it turns out that the resultant equation of motion for the scalar field still remains at the second order as the higher order derivative terms cancel away. It is one of the possible scalar field models in curved space time that contains higher order terms in the Lagrangian, but still maintains a 
second order equation for both metric and the field [20], similar to the Gauss-Bonnet term in the gravity action [21].

It should be noted that a Galilean field corresponds to those class of scalar field models which are invariant in a Minkowski space time under the Galilean type field transformation, viz. $\phi \rightarrow \phi+b_{\mu} x^{\mu}+c$, where $c$ is a constant and $b_{\mu}$ is a constant vector [22, 23]. Note that the transformation, $\phi \rightarrow \phi+b_{\mu} x^{\mu}+c$, corresponds to shifting the field derivative by a constant vector $b_{\mu}$ similar to the standard Galilean transformation $\dot{\vec{x}} \rightarrow \dot{\vec{x}}+\vec{V}$ in particle mechanics. One of the type of scalar field model admitting this type of invariance has Lagrangian of the form $\mathcal{L}_{3} \propto X \square \phi$ [24]. The Lagrangian of the G-inflation field contains the term $G(X, \phi) \square \phi$ which can be viewed as the generalization of this type of Galilean interaction, although a generic $G(X, \phi)$ may not admit invariance under $\partial_{\mu} \phi \rightarrow \partial_{\mu} \phi+b_{\mu}$. Nevertheless, these scalar fields are dubbed as Galilean fields.

The three class of models of inflation discussed above can be characterized by observables such as $n_{S}, n_{T}$ and $r$, where $\left\{n_{S}, n_{T}\right\}$ are the spectral indices for scalar and tensor perturbations, respectively, and $r$ is the tensor-to-scalar ratio $[25,26]$. For the first two classes of inflation models discussed above, namely, the canonical and the non-canonical scalar field models, it turns out that $r$ and $n_{T}$ satisfy the following consistency relation $r \leq-8 n_{T}$ [27]. This is, in fact, the consequence of the subluminal propagation ${ }^{1}$ of the scalar field perturbations. In models which lead to superluminal speed of sound ${ }^{2}$, one gets $r>-8 n_{T}$, see for instance [28]. However, it is recently demonstrated that for the G-inflation models [19, 30], this consistency relation can be violated even after ensuring that the speed of sound of scalar field perturbations is subluminal. It is therefore interesting to investigate whether the subluminal propagation of scalar field perturbations in G-inflation models put any upper limit on the ratio $r /\left(8\left|n_{T}\right|\right)$. For the case of canonical and non-canonical scalar field models of inflation, it is known that the upper limit of the above mentioned ratio is unity. However, it is not known whether such an upper limit exists for G-inflation models.

To address this issue, we consider a restricted class of analytically solvable G-inflation model in which the $K(X, \phi)$ term only contributes the potential $V(\phi)$ and $G(X, \phi) \propto X^{n}$ and it is independent of the field $\phi$. This will ensure that the contribution of the k-inflation term $K(X, \phi)$ is minimal and any violation of the consistency relation can therefore be attributed to the G-inflation term $G(X, \phi)$. For this class of model, we obtain the form of the potential $V(\phi)$ which can lead to power law inflation. For power law solution, it is possible to arrive at an exact inflationary consistency relation between $r$ and $n_{T}$ without imposing the slow roll condition. We therefore derive such a consistency relation for the class of G-inflation model driving power law inflation. The limit on the ratio $r /\left(8\left|n_{T}\right|\right)$ can thus be found by imposing the restriction that the speed of sound for scalar field perturbations is subluminal.

This paper is organised as follows. In Sec. 2, all the basic equations describing the field dynamics of a generic G-inflation model in a spatially flat universe is discussed. In Sec. 3, we introduce a specific class of G-inflation model which can drive power law inflation. The inflationary consistency relation for such a power law model is derived Sec. 4. In Sec. 5, the observationally viable Galilean inflationary scenario is compared with those based on noncanonical scalar field settings. Finally, the main results of this paper are highlighted in Sec. 6. The derivation of the expression for the speed of sound in G-inflation models is described in

\footnotetext{
${ }^{1}$ It is also possible to violate the consistency relation $r \leq-8 n_{T}$ in standard canonical inflation models, even in the absence of superluminal propagations, if additional fields generates perturbations [31].

${ }^{2}$ The question of whether or not superluminal propagation of scalar field perturbations violates causality is debated in the literature [29].
} 
Appendix A. Throughout this paper, we shall adopt the metric signature of $(+---)$ and we express every equations in natural units thereby setting $\hbar=c=1$. In such units the reduced Planck mass $M_{p}$ is defined as $M_{p}=(8 \pi G)^{-1 / 2}$.

\section{G-Inflation Preliminaries}

We consider the following Einstein-Hilbert action with a Galilean scalar field:

$$
\mathcal{S}=-\frac{M_{p}^{2}}{2} \int R \sqrt{-g} \mathrm{~d}^{4} x+\int \sqrt{-g} \mathrm{~d}^{4} x \mathcal{L}(\phi, X, \square \phi)
$$

where

$$
\mathcal{L}(\phi, X, \square \phi)=K(X, \phi)+G(X, \phi) \square \phi,
$$

is the Lagrangian density of the G-inflation field $[18,19]$. In the above Lagrangian the function $K(X, \phi)$ and $G(\phi, X)$ can, in general, be an arbitrary function of the field $\phi$ and the kinetic term $X=(1 / 2) \partial_{\mu} \phi \partial^{\mu} \phi$. The form of the Lagrangian (2.2) takes care of almost all minimally coupled scalar field models of inflation. When $G(X, \phi)=0$, the model represent non-canonical scalar field inflation also known as k-inflation ${ }^{3}[14,15]$ and in addition if $K(X, \phi)=X-V(\phi)$ it reduces to the standard canonical scalar field model of inflation.

The Lagrangian (2.2) contains the second derivative of the field $\phi$. After removing the boundary term $\left(G \partial^{\mu} \phi\right)_{; \mu}$ one gets the following equivalent Lagrangian density [18]:

$$
\mathcal{L}^{(\mathrm{e})}=K-2 X G_{\phi}-G_{X} \partial^{\mu} X \partial_{\mu} \phi,
$$

where the notations such as $G_{\phi}$ denotes $\partial G / \partial \phi$. Note that when $G_{X}=0$, the above Lagrangian is a function only of $X$ and $\phi$, and hence in this scenario it is equivalent to a k-inflation model. However, when $G_{X} \neq 0$, the Lagrangian (2.2) contains the second order derivative in $\phi$ after removing the boundary term and hence, in this case the model is phenomenologically distinct from the k-inflation models. We will be considering such a case where $G_{X} \neq 0$.

From the action (2.1), the field equation for $\phi$ is given by

$$
\frac{\partial}{\partial \phi}(\mathcal{L} \sqrt{-g})-\partial_{\mu}\left(\frac{\partial \mathcal{L} \sqrt{-g}}{\partial\left(\partial_{\mu} \phi\right)}\right)+\partial_{\mu} \partial_{\nu}\left(\frac{\partial \mathcal{L} \sqrt{-g}}{\partial\left(\partial_{\mu} \partial_{\nu} \phi\right)}\right)=0
$$

On substituting the Lagrangian density (2.2) in the above equation, we get

$$
\begin{gathered}
\left(2 G_{\phi}-2 X G_{X \phi}-K_{X}\right) \square \phi+\left(2 G_{X \phi}-K_{X X}\right) \partial^{\mu} \phi \partial_{\mu} X+2 X\left(G_{\phi \phi}-K_{X \phi}\right)+K_{\phi}+ \\
G_{X}\left[\left(\partial^{\mu} \phi\right)_{; \nu}\left(\partial^{\nu} \phi\right)_{; \mu}-(\square \phi)^{2}+R_{\mu \nu} \partial^{\mu} \phi \partial^{\nu} \phi\right]+G_{X X}\left[\partial^{\mu} X \partial_{\mu} X-\left(\partial^{\mu} \phi \partial_{\mu} X\right) \square \phi\right]=0
\end{gathered}
$$

Note that one gets the same equation of motion if instead of $\mathcal{L}$ from Eq. (2.2) one substitutes the equivalent Lagrangian (2.3) in Eq. (2.4). It is also important to note that although we started with the action (2.1) in which the field $\phi$ is minimally coupled to gravity, the resulting field equation contains a term $R_{\mu \nu} \partial^{\mu} \phi \partial^{\nu} \phi$ indicating a coupling between the Ricci tensor

\footnotetext{
${ }^{3}$ The class of models with $\mathcal{L}=\mathcal{L}(\phi, X)$ are known as k-inflation or kinetic inflation since in some of these models, first introduced in Ref. $[14,15]$, it is the kinetic term in the Lagrangian which drives inflation. Therefore, in the k-inflation models described in Ref. $[14,15], \mathcal{L}(X, \phi) \rightarrow 0$ as $X \rightarrow 0$. However, not all non-canonical models satisfies this criteria, see for instance Refs. [16, 40, 41]. Nevertheless, a generic model with $\mathcal{L}=\mathcal{L}(X, \phi)$, may be referred as either k-inflation or as non-canonical model of inflation.
} 
and the kinetic term. It is for this reason, these class of models are also known as kinetic gravity braiding models [18].

On varying the action (2.1) with respect to the metric $g_{\mu \nu}$ gives the Einstein's equation $G_{\mu \nu}=(8 \pi G) T_{\mu \nu}$, where the energy momentum tensor $T_{\mu \nu}$ is defined as

$$
T_{\mu \nu}=\frac{2}{\sqrt{-g}}\left[\frac{\partial}{\partial g^{\mu \nu}}(\mathcal{L} \sqrt{-g})-\partial_{\alpha}\left(\frac{\partial \mathcal{L} \sqrt{-g}}{\partial\left(g^{\mu \nu}, \alpha\right.}\right)\right] .
$$

Substituting $\mathcal{L}$ from Eq. $(2.2)$ or $\mathcal{L}^{(\mathrm{e})}$ from Eq. (2.3) in the above equation gives

$$
\begin{aligned}
T^{\mu}{ }_{\nu}= & -G_{X}\left[\partial^{\mu} \phi \partial_{\nu} X+\partial^{\mu} X \partial_{\nu} \phi\right]-\left[2 G_{\phi}-G_{X} \square \phi-K_{X}\right] \partial^{\mu} \phi \partial_{\nu} \phi \\
& +\left[-K+G_{X} \partial^{\alpha} X \partial_{\alpha} \phi+2 X G_{\phi}\right] \delta_{\nu}^{\mu} .
\end{aligned}
$$

Considering a spatially flat Friedmann-Robertson-Walker (FRW) universe described by the line element

$$
\mathrm{d} s^{2}=\mathrm{d} t^{2}-a^{2}(t)\left[\mathrm{d} x^{2}+\mathrm{d} y^{2}+\mathrm{d} z^{2}\right]
$$

the above expression (2.7) for the energy momentum tensor takes the diagonal form:

$$
T_{\nu}^{\mu}=\operatorname{dia}\left\{\rho_{\phi},-p_{\phi},-p_{\phi},-p_{\phi}\right\},
$$

where the energy density $\rho_{\phi}$ and the pressure $p_{\phi}$ are given by

$$
\begin{aligned}
& \rho_{\phi}=2 X K_{X}-K-2 X G_{\phi}+6 H \dot{\phi} X G_{X}, \\
& p_{\phi}=K-2 X G_{\phi}-2 X \ddot{\phi} G_{X} .
\end{aligned}
$$

In the above two equations, $H \equiv \dot{a} / a$, where $a(t)$ is the scale factor. The Einstein's equation $G_{\mu \nu}=(8 \pi G) T_{\mu \nu}$ implies that the scale factor $a(t)$ satisfies the following Friedmann equations:

$$
\begin{aligned}
\left(\frac{\dot{a}}{a}\right)^{2} & =\left(\frac{8 \pi G}{3}\right) \rho_{\phi}, \\
\frac{\ddot{a}}{a} & =-\left(\frac{4 \pi G}{3}\right)\left(\rho_{\phi}+3 p_{\phi}\right),
\end{aligned}
$$

Note that the expression for the energy density $\rho_{\phi}$, as described in Eq. (2.10), contains $H$. Therefore, the first Friedmann equation (2.12) describes a quadratic equation for $H$ unlike the usual case when one considers canonical or non-canonical scalar field models. To ensure that $H$ is real and positive definite, it is necessary that the following conditions are satisfied:

$$
\begin{gathered}
\frac{\dot{\phi} X G_{X}}{M_{p}^{2}} \geq 0 \\
\left(\frac{\dot{\phi} X G_{X}}{M_{p}^{2}}\right)^{2} \geq\left(\frac{1}{3 M_{p}^{2}}\right)\left(K-2 X K_{X}+2 X G_{\phi}\right)
\end{gathered}
$$

With the above condition, the Friedmann equation (2.12) becomes

$$
H=\frac{\dot{\phi} X G_{X}}{M_{p}^{2}}+\left(\frac{1}{M_{p}^{2}}\right)\left[\left(\dot{\phi} X G_{X}\right)^{2}+\left(\frac{M_{p}^{2}}{3}\right)\left(2 X K_{X}-K-2 X G_{\phi}\right)\right]^{1 / 2}
$$


Moving on to the field equation for $\phi$ as described in Eq. (2.5), notice that because of the term $R_{\mu \nu} \partial^{\mu} \phi \partial^{\nu} \phi$, the equation of motion for $\phi$ contains terms proportional to $\dot{H}$. This can be eliminated using the two Friedmann equations (2.12) and (2.13). The equation of motion for $\phi$ can then be expressed as

$$
C_{1} \ddot{\phi}+C_{2}(3 H \dot{\phi})+2 X C_{3}-K_{\phi}=0
$$

where

$$
\begin{aligned}
& C_{1}=K_{X}+2 X K_{X X}-2 G_{\phi}-2 X G_{X \phi}+6 H \dot{\phi}\left(G_{X}+X G_{X X}\right)+\frac{6 X^{2} G_{X}^{2}}{M_{p}^{2}}, \\
& C_{2}=K_{X}-2 G_{\phi}+2 X G_{X \phi}+3 H \dot{\phi} G_{X}-\frac{6 X^{2} G_{X}^{2}}{M_{p}^{2}} \\
& C_{3}=K_{X \phi}-G_{\phi \phi}-\left(\frac{3 X G_{X}}{M_{p}^{2}}\right)\left(K_{X}-2 G_{\phi}\right) .
\end{aligned}
$$

Eqs. (2.16) and (2.17) forms the two closed set of equations describing the evolution of $a(t)$ and $\phi(t)$. For the case of canonical scalar field which corresponds to choosing $K(X, \phi)=$ $X-V(\phi)$ and $G(X, \phi)=0$, the field equation (2.17) reduces to the standard Klein-Gordon equation viz. $\ddot{\phi}+3 H \dot{\phi}+V_{\phi}=0$.

\section{Power law G-Inflation}

Our aim in this paper is to derive an exact consistency relation in G-inflation models without assuming slow roll. It is possible to do so in the case of power law inflation for which one can obtain an exact analytical expression for $r$ and $n_{T}$. For the case of kinetic power law inflation, such an exact consistency relation is derived in Ref. [14]. It follows from the analysis of Ref. [14] that $r \leq-8 n_{T}$ when the speed of sound is subluminal.

Although, G-inflation corresponds to a wider class of models with generic $K(X, \phi)$ and $G(X, \phi)$ in the Lagrangian (2.2), to understand the exact reason for the violation of the consistency relation as noted in Ref. [30], it is necessary to minimize the contribution from the k-inflation term $K(X, \phi)$. If we eliminate the contribution of the $K(X, \phi)$ altogether, and chose $G(X, \phi) \propto X^{n}$, then it lead to $c_{s}^{2}<0$ thereby making the system violently unstable. This also happens when $K(X, \phi)=0$ and $G(X, \phi)=g(\phi) X^{n}$ with an integer value for $n$. For this reason we restrict ourself to a subclass of these models with $K(X, \phi)=-V(\phi)$ and $G(X, \phi) \propto X^{n}$. The Lagrangian of this restricted class of G-inflation model considered in this paper is therefore given by:

$$
\mathcal{L}(\phi, X, \square \phi)=\frac{X^{n} \square \phi}{M^{4 n-1}}-V(\phi),
$$

where $n$ and $M$ are parameters of the model with $n$ being dimensionless and $M$ has dimensions of mass. We will now obtain the form of the potential $V(\phi)$ which can drive power law inflation wherein the scale factor evolves as

$$
a(t) \propto t^{q} ; \quad q>1
$$


For model (3.1), the energy density $\rho_{\phi}$ and the pressure $p_{\phi}$ turns out to be:

$$
\begin{aligned}
& \rho_{\phi}=\left(\frac{3 n H}{2^{n-1}}\right)\left(\frac{\dot{\phi}^{2 n+1}}{M^{4 n-1}}\right)+V(\phi), \\
& p_{\phi}=-\left(\frac{n \ddot{\phi}}{2^{n-1}}\right)\left(\frac{\dot{\phi}^{2 n}}{M^{4 n-1}}\right)-V(\phi) .
\end{aligned}
$$

When $a(t) \propto t^{q}$, the Friedmann equations (2.12) and (2.13) implies that

$$
\begin{aligned}
& \rho_{\phi}=\frac{3 M_{p}^{2} q^{2}}{t^{2}}, \\
& p_{\phi}=w \rho_{\phi},
\end{aligned}
$$

where $w=2 /(3 q)-1$. From Eqs. (3.2) to (3.5), it follows that

$$
\begin{gathered}
\frac{M_{p}^{2}}{t}=\left(\frac{3 n \dot{\phi}^{2 n+1}}{2^{n} M^{4 n-1}}\right)\left(1-\frac{\ddot{\phi}}{3 H \dot{\phi}}\right), \\
V(\phi)=-\left(\frac{n 2^{2-n}}{1+w}\right)\left(\frac{\dot{\phi}^{2 n+1}}{M^{4 n-1}}\right)\left(\frac{1}{t}\right)+\frac{4 M_{p}^{2}}{3(1+w)^{2} t^{2}} .
\end{gathered}
$$

It can be verified that Eq. (3.6) admit solution of the form:

$$
\phi(t)=A M_{p}^{\beta+1} t^{\beta} ; \quad \text { where } \beta=\frac{2 n}{2 n+1},
$$

and

$$
A=\left(\frac{2 n+1}{2 n}\right)\left\{\left(\frac{2^{n}}{3 n}\right)\left(\frac{2(2 n+1)}{2(2 n+1)+(1+w)}\right)\left(\frac{M}{M_{p}}\right)^{4 n-1}\right\}^{\frac{1}{2 n+1}} .
$$

Substituting the solution (3.8) in Eq. (3.7), we get the following form of the potential:

$$
V(\phi)=\frac{V_{0}}{\left(\phi / M_{p}\right)^{s}} ; \quad \text { where } \quad s=\frac{2 n+1}{n},
$$

and

$$
V_{0}=\left(\frac{4 A^{s} M_{p}^{4}}{3(1+w)^{2}}\right)\left(\frac{1-(4 n+1) w}{2(2 n+1)+(1+w)}\right) .
$$

In the model (3.1) with the above form of the potential, although tedious, it is straight forward to verify that the solution (3.8) with $a(t) \propto t^{q}$ satisfy both the Friedmann's equations (2.12), (2.13) and the scalar field equation (2.17). Hence, in the Lagrangian (3.1), an inverse power law potential of the form (3.10) can drive power law inflation with $a(t) \propto t^{q}$. It is interesting to note that such inverse power law potentials also drive power law inflation in non-canonical scalar field models, see for instance power law models described in Refs. [14, 15, 32]. However, in the case canonical scalar field driven inflation, an inverse power law potential leads to intermediate inflation [33, 34]. 


\section{Consistency relation in Galilean Power law inflation}

In this section we shall derive the consistency relation for the Galilean power law inflation model described in the preceding section. However, before moving on the specific model (3.1), let us first consider the generic G-inflation scenario. To obtain the scalar and tensor perturbations generated by the inflation field, we consider the following FRW line element with metric perturbations [35-37]

$$
\mathrm{d} s^{2}=(1+2 A) \mathrm{d} t^{2}-2 a(t)\left(\partial_{i} B\right) \mathrm{d} t \mathrm{~d} x^{i}-a^{2}(t)\left[(1-2 \psi) \delta_{i j}+2\left(\partial_{i} \partial_{j} E\right)+h_{i j}\right] \mathrm{d} x^{i} \mathrm{~d} x^{j}
$$

where $A, B, \psi$ and $E$ are scalar degree of metric perturbation and $h_{i j}$ is the tensor perturbations. The vector perturbations are ignored as it is known that scalar fields do not lead to such perturbations. The perturbation in the scalar field is defined as

$$
\phi(\vec{x}, t)={ }^{(0)} \phi(t)+\delta \phi(\vec{x}, t)
$$

where ${ }^{(0)} \phi(t)$ is the background field which, for the G-inflation case, satisfies Eq. (2.17). The perturbation $\delta \phi(\vec{x}, t)$ being a gauge dependent quantity, one generally introduce the following gauge invariant quantity $\mathcal{R}$ known as curvature perturbation:

$$
\mathcal{R} \equiv \psi+\left(\frac{H}{\dot{\phi}}\right) \delta \phi .
$$

In the generic model with the Lagrangian (2.2), the second order action for the curvature perturbation $\mathcal{R}$ turns out to be $[18,19,38]$

$$
\mathcal{S}^{(2)}=\frac{1}{2} \int \mathrm{d}^{3} x \mathrm{~d} \eta z^{2}\left[\mathcal{R}^{\prime 2}-c_{s}^{2}\left(\partial_{i} \mathcal{R}\right)^{2}\right]
$$

where $\eta$ is the conformal time and $c_{s}$ is the speed of sound for the G-inflation field whose square is given by

$$
c_{s}^{2} \equiv \frac{K_{X}-2 G_{\phi}+2 X G_{X \phi}+2 \ddot{\phi}\left(G_{X}+X G_{X X}\right)+4 H \dot{\phi} G_{X}-2 X^{2} G_{X}^{2} / M_{p}^{2}}{K_{X}+2 X K_{X X}-2 G_{\phi}-2 X G_{X \phi}+6 H \dot{\phi}\left(G_{X}+X G_{X X}\right)+6 X^{2} G_{X}^{2} / M_{p}^{2}} .
$$

In the action (4.3), the function $z$ is defined as

$$
z \equiv\left(\frac{a \dot{\phi} \sqrt{\mathcal{F}}}{c_{s} H}\right)\left[1-\left(\frac{\dot{\phi}^{3} G_{X}}{2 H M_{p}^{2}}\right)\right]^{-1}
$$

where

$$
\mathcal{F}=K_{X}-2 G_{\phi}+2 X G_{X \phi}+2 \ddot{\phi}\left(G_{X}+X G_{X X}\right)+4 H \dot{\phi} G_{X}-\frac{2 X^{2} G_{X}^{2}}{M_{p}^{2}} .
$$

From the action (4.3), one gets the following equation of motion for the curvature perturbation

$$
\mathcal{R}_{k}^{\prime \prime}+2\left(\frac{z^{\prime}}{z}\right) \mathcal{R}_{k}^{\prime}+c_{s}^{2} k^{2} \mathcal{R}_{k}=0,
$$

where $\mathcal{R}_{k}$ is the amplitude of the curvature perturbation $\mathcal{R}$ in the Fourier space and $k$ is the wavenumber. Note that just like in the case of canonical or non-canonical scalar field 
driven inflation, the curvature perturbation $\mathcal{R}$ is also conserved at the superhorizon scales in G-inflation models, for a proof see Ref. [39].

In terms of the Mukhanov-Sasaki variable $u_{k}=z \mathcal{R}_{k}$, Eq. (4.7) becomes:

$$
u_{k}^{\prime \prime}+\left(c_{s}^{2} k^{2}-\frac{z^{\prime \prime}}{z}\right) u_{k}=0 .
$$

This is exactly identical to the corresponding equation for the k-inflation field [14], the difference being $c_{s}^{2}$ and $z$ in the above equation are different from those that appear in the Mukhanov-Sasaki equation in k-inflation models (see Eq. (28) in Ref. [14]).

Similarly, for tensor perturbations one gets the following equation:

$$
v_{k}^{\prime \prime}+\left(k^{2}-\frac{a^{\prime \prime}}{a}\right) v_{k}=0,
$$

where $v_{k}=a h_{k}\left(M_{p} / 2\right)$, with $h_{k}$ being the Fourier amplitude of tensor perturbations. Unlike the case of Mukhanov-Sasaki equation for the scalar variable $u_{k}$, the above equation is identically valid for all minimally coupled scalar field models of inflation since tensor perturbations evolves independent of the scalar perturbations at the linear order.

The scalar and tensor power spectrum are defined as

$$
\begin{gathered}
\mathcal{P}_{S}(k) \equiv\left(\frac{k^{3}}{2 \pi^{2}}\right)\left|\mathcal{R}_{k}\right|^{2}=\left(\frac{k^{3}}{2 \pi^{2}}\right)\left(\frac{\left|u_{k}\right|}{z}\right)^{2}, \\
\mathcal{P}_{T}(k) \equiv 2\left(\frac{k^{3}}{2 \pi^{2}}\right)\left|h_{k}\right|^{2}=2\left(\frac{k^{3}}{2 \pi^{2}}\right)\left(\frac{4}{M_{p}^{2}}\right)\left(\frac{\left|v_{k}\right|}{a}\right)^{2} .
\end{gathered}
$$

Furthermore, one defines the scalar and tensor spectral index as:

$$
\begin{aligned}
n_{S}-1 & \equiv \frac{\mathrm{d} \ln \mathcal{P}_{S}}{\mathrm{~d} \ln k}, \\
n_{T} & \equiv \frac{\mathrm{d} \ln \mathcal{P}_{T}}{\mathrm{~d} \ln k} .
\end{aligned}
$$

And finally the tensor-to-scalar ratio $r$ is defined as

$$
r \equiv \frac{\mathcal{P}_{T}}{\mathcal{P}_{S}} .
$$

It is clear from Eq. (4.8) that it is the function $z$ which can lead to a different evolution for the mode function $u_{k}$ in a G-inflation model from those in a k-inflation model which has the same value for the speed of sound for scalar perturbations. It is therefore illustrative to express the function $z$ defined in Eq. (4.5) as

$$
z=\frac{\tilde{z}}{\lambda}
$$

where $\tilde{z}$, defined as

$$
\tilde{z} \equiv \frac{a\left(\rho_{\phi}+p_{\phi}\right)^{1 / 2}}{c_{s} H},
$$


is the one that appears in the Mukhanov-Sassaki equation for the k-inflation field [14] and $\lambda$ is defined as

$$
\lambda=\left[1-\left(\frac{\dot{\phi}^{3} G_{X}}{2 H M_{p}^{2}}\right)\right] \sqrt{\frac{\mathcal{Q}}{2 X \mathcal{F}}},
$$

where $\mathcal{F}$ is defined in Eq. (4.6) and $\mathcal{Q}$ is given by

$$
\mathcal{Q}=2 X K_{X}-4 X G_{\phi}-2 X G_{X}(\ddot{\phi}-3 H \dot{\phi}) .
$$

Note that in the case of k-inflation which corresponds to setting $G(X, \phi)=0$, in the Lagrangian (2.2), one gets $\lambda=1$. Hence, it is because of this $\lambda$ factor in Eq. (4.15) that makes the scalar power spectrum in G-inflation different from those in an equivalent kinflation model which leads to the same background evolution and has the same value for $c_{s}^{2}$. Since Eq. (4.9) is valid for all minimally coupled scalar field models of inflation, the tensor power spectrum in G-inflation is exactly the same as those in an equivalent k-inflation model which leads to the same background evolution. It is for this reason the tensor-to-scalar ratio in G-inflation will be different from those in an equivalent k-inflation model. This point will be illustrated in detail for the power law model considered in the preceding section

For the restricted class of G-inflation model (3.1) with an inverse power law potential (3.10) driving power law inflation with $a(t) \propto t^{q}$, it follows from Eqs. (3.8) and (4.4) that

$$
c_{s}^{2}=\left(\frac{1}{3(2 n+1)}\right)\left\{\frac{[4(2 n+1)-3 n(1+w)][2(2 n+1)+(1+w)]-(2 n+1)^{2}(1+w)}{4 n(2 n+1)+(1+w)(4 n+1)}\right\},
$$

where $w$ is the equation of state parameter which is related to parameter $q$ in the power law solution $a(t) \propto t^{q}$ as $w=-1+2 /(3 q)$. It is clear from Eq. (4.19) that the speed of sound is identically constant for the Galilean power law inflation model (3.1) and in the slow roll limit which corresponds to $(1+w) \ll 1$ or equivalently $q \gg 1$, one gets $c_{s}^{2} \simeq 2 /(3 n)$. When $n=1$, the slow roll value of $c_{s}^{2}$ is $2 / 3$ and this is consistent with Ref. [30] which considered Higgs G-inflation. In the left panel of Fig. $1, c_{s}^{2}$ is plotted as a function of $w$. Note that the speed of sound for the Galilean model is subluminal [51]. Furthermore, the solution (3.8) also implies that the $\lambda$ parameter defined in Eq. (4.17) is a constant and is given by

$$
\lambda=\left\{\frac{6[(2 n+1)-n(1+w)]^{2}}{8(2 n+1)^{2}-(1+w)\left[16 n^{2}+2 n-3\right]-3 n(1+w)^{2}}\right\}^{1 / 2} .
$$

The parameter $\lambda$ is plotted as a function of $w$ in the right panel of Fig. 1. In the slow roll limit which corresponds to $(1+w) \ll 1$, the parameter $\lambda \rightarrow \sqrt{3 / 4}$ irrespective of the value of $n$ in the Lagrangian (3.1).

For the power law solution $a(t) \propto t^{q}$, the function $z$ defined in Eq. (4.15) becomes

$$
z=\left(\frac{M_{p} a}{\lambda c_{s}}\right) \sqrt{\frac{2}{q}} .
$$

With $z$ given by the above expression, the Mukhanov-Sasaki equation (4.8) can be expressed as

$$
u_{k}^{\prime \prime}+\left(c_{s}^{2} k^{2}-\frac{\nu^{2}-(1 / 4)}{\eta^{2}}\right) u_{k}=0
$$



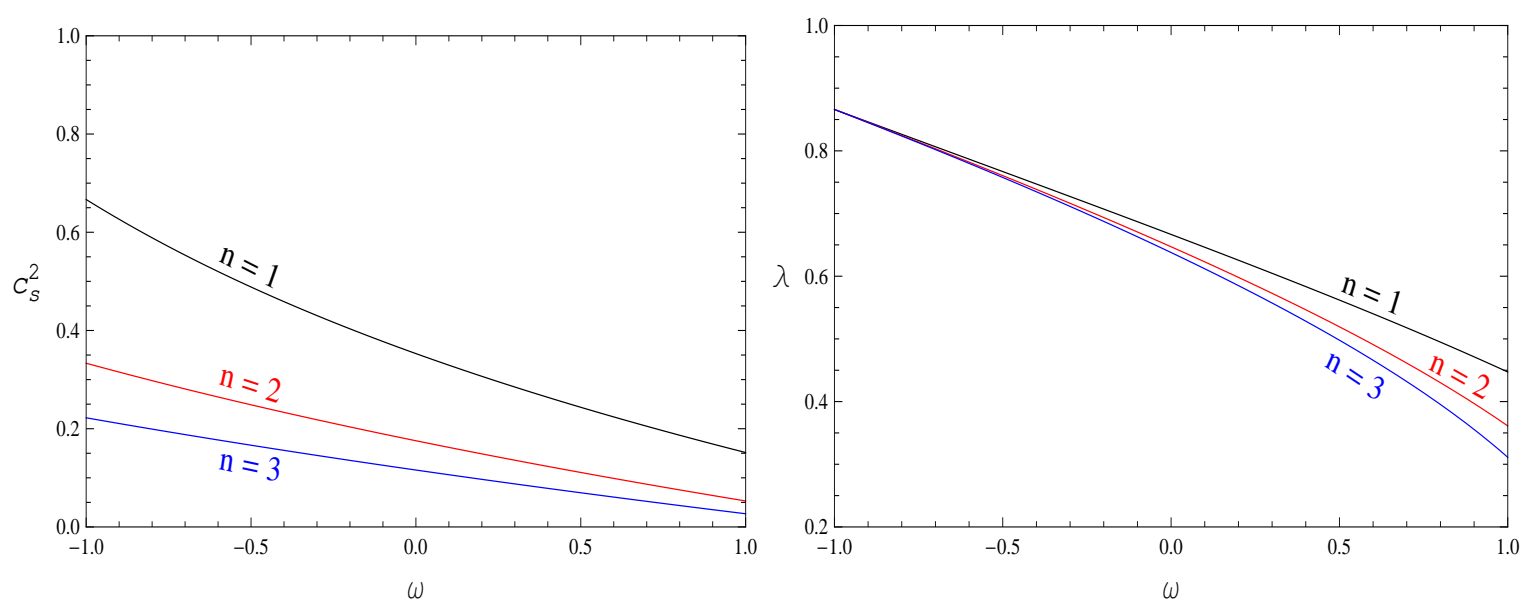

Figure 1. In the left panel, $c_{s}^{2}$ for the Galilean power law inflation model, as given in Eq. (4.19), is plotted as a function of equation of state parameter $w$ for different values of $n$ in the Lagrangian (3.1). In the right panel $\lambda$ given in Eq. (4.20) is plotted as a function of $w$. Note that both $c_{s}^{2}$ and $\lambda$ are positive definite and they are less than unity. In this figure, the range of $w$ is from -1 to +1 , although for inflation one only need to consider the regime where $w<-1 / 3$.

where

$$
\nu=\frac{3 q-1}{2(q-1)} .
$$

The general solution of the above equation can be expressed as

$$
u_{k}=\sqrt{-c_{s} k \eta}\left[\mathrm{C}_{1} \mathrm{H}_{\nu}^{(1)}\left(-c_{s} k \eta\right)+\mathrm{C}_{2} \mathrm{H}_{\nu}^{(2)}\left(-c_{s} k \eta\right)\right],
$$

where $\mathrm{C}_{1}$ and $\mathrm{C}_{2}$ are constants of integration, $\mathrm{H}_{\nu}^{(1)}(x)$ and $\mathrm{H}_{\nu}^{(2)}(x)$ are Hankel functions of first and second kind, respectively. On imposing the Bunch-Davis initial condition that $u_{k}=\left(2 k c_{s}\right)^{-(1 / 2)} \exp \left[-i c_{s} k \eta\right]$ at the sub-horizon scales $\left(-c_{s} k \eta \gg 1\right)$ leads to $\mathrm{C}_{2}=0$ and the above solution for the mode function $u_{k}$ becomes

$$
u_{k}=\sqrt{\frac{-\pi \eta}{4}} \exp \left[i\left(\frac{\pi}{2}\right)\left(\nu+\frac{1}{2}\right)\right] \mathrm{H}_{\nu}^{(1)}\left(-c_{s} k \eta\right)
$$

Similarly for the tensor perturbations, the solution of the Eq. (4.9), satisfying the BunchDavis initial condition turns out to be identical to $u_{k}$, except for tensor perturbations $c_{s}=1$. Therefore,

$$
v_{k}=\sqrt{\frac{-\pi \eta}{4}} \exp \left[i\left(\frac{\pi}{2}\right)\left(\nu+\frac{1}{2}\right)\right] \mathrm{H}_{\nu}^{(1)}(-k \eta) .
$$

From the above solutions for mode functions $u_{k}$ and $v_{k}$, the scalar and tensor power spectrum defined in Eqs. (4.10) and (4.11), respectively, at the super horizon scales $\left(-c_{s} k \eta \ll 1\right)$ turns out to be

$$
\begin{aligned}
& \mathcal{P}_{S}(k)=A_{S} k^{-2 /(q-1)}, \\
& \mathcal{P}_{T}(k)=A_{T} k^{-2 /(q-1)},
\end{aligned}
$$


where

$$
\begin{aligned}
& A_{S}=\left(\frac{q \lambda^{2}}{16 \pi^{3} c_{s}^{2 \nu-2}}\right)\left(\frac{\Gamma(\nu) 2^{\nu}}{M_{p} a_{1}}\right)^{2}, \\
& A_{T}=\left(\frac{1}{\pi^{3}}\right)\left(\frac{\Gamma(\nu) 2^{\nu}}{M_{p} a_{1}}\right)^{2} .
\end{aligned}
$$

In the above equations $\Gamma(\nu)$ is Gamma function and $a_{1}$ is a constant that appears in the equation describing the evolution of scalar factor in conformal time, viz. $a(\eta)=a_{1}[-\eta]^{-q /(q-1)}$. The value of this parameter $a_{1}$ in Eqs. (4.27) and (4.28) can be fixed using the CMB normalization, namely, $P_{S}\left(k_{*}\right)=2.2 \times 10^{-9}$ at the pivot scale $k_{*}=0.05 \mathrm{Mpc}^{-1}$ [4]. For $n=4$ and $q=60$, it turns out that $a_{1} \simeq 4.4 \times 10^{5} M_{p}^{-q /(q-1)}$. Since $a(\eta)=a_{1}[-\eta]^{-q /(q-1)}$ implies that $a(t)=a_{*}\left(t / t_{*}\right)^{q}$, it turns out that $a_{*}=a_{1}^{1-q}\left[t_{*} /(q-1)\right]^{q}$. Let $t_{*}=q / H_{*}$ be the time at which the pivot scale exit the cosmological horizon $\left(a_{*} H_{*}=k_{*}\right)$, which in turn implies that

$$
H_{*}=\frac{1}{a_{1}}\left[\left(\frac{1}{k_{*}}\right)\left(\frac{q}{q-1}\right)^{q}\right]^{\frac{1}{q-1}} .
$$

Therefore, the CMB normalized value of $a_{1}$ can be used to determined the value of the Hubble parameter $H_{*}$ when the pivot scale exit the cosmological horizon. For $n=4$ and $q=60$, we find that $H_{*}=2.2 \times 10^{-5} M_{p}$.

From Eqs. (4.25) and (4.26), the scalar and tensor spectral index, defined in Eqs. (4.12) and (4.13), respectively, turns out to be

$$
n_{S}-1=n_{T}=-\frac{2}{q-1} .
$$

This is exactly the same for the case of standard power law inflation driven by a canonical scalar field with an exponential potential [44-46] and one also gets the same $n_{S}$ and $n_{T}$ in the power law scenario in some non-canonical scalar field models such as those discussed in Refs. [14, 15, 32]. In fact, Eq. (4.29) is valid for any model of power law inflation based on the Lagrangian (2.2) but for which the parameters $c_{s}^{2}$ and $\lambda$ defined in Eqs. (4.4) and (4.17), respectively, are identically constant. This simply follows from the fact that whenever $c_{s}^{2}$ and $\lambda$ are constant, the solution (4.23) for the mode function $u_{k}$ satisfy the Mukhanov Sasaki equation (4.8) during power law expansion and the resultant $n_{S}$ (and $n_{T}$ ) is the consequence of this solution for $u_{k}$ (and $v_{k}$ ).

Eqs. (4.25) and (4.26) lead to the following tensor-to-scalar ratio

$$
r=\left(\frac{16}{q \lambda^{2}}\right)\left(\frac{1}{c_{s}}\right)^{\frac{1+q}{1-q}} .
$$

It is evident from the above expression that unlike the spectral indices $n_{S}$ and $n_{T}$ which depends only on the value of $q$ in the power law solution $a(t) \propto t^{q}$, the tensor-to-scalar ratio $r$ also depends on the model parameters $c_{s}^{2}$ and $\lambda$ defined in Eqs. (4.4) and (4.17), respectively. Therefore, $r$ contains the details of the dynamics of inflation and it can play an important role in distinguishing models of inflation [47].

Eqs. (4.29) and (4.30) imply the following consistency relation

$$
r=-\left(\frac{8 n_{T}}{\lambda^{2}}\right)\left(\frac{q-1}{q}\right)\left(\frac{1}{c_{s}}\right)^{\frac{1+q}{1-q}},
$$



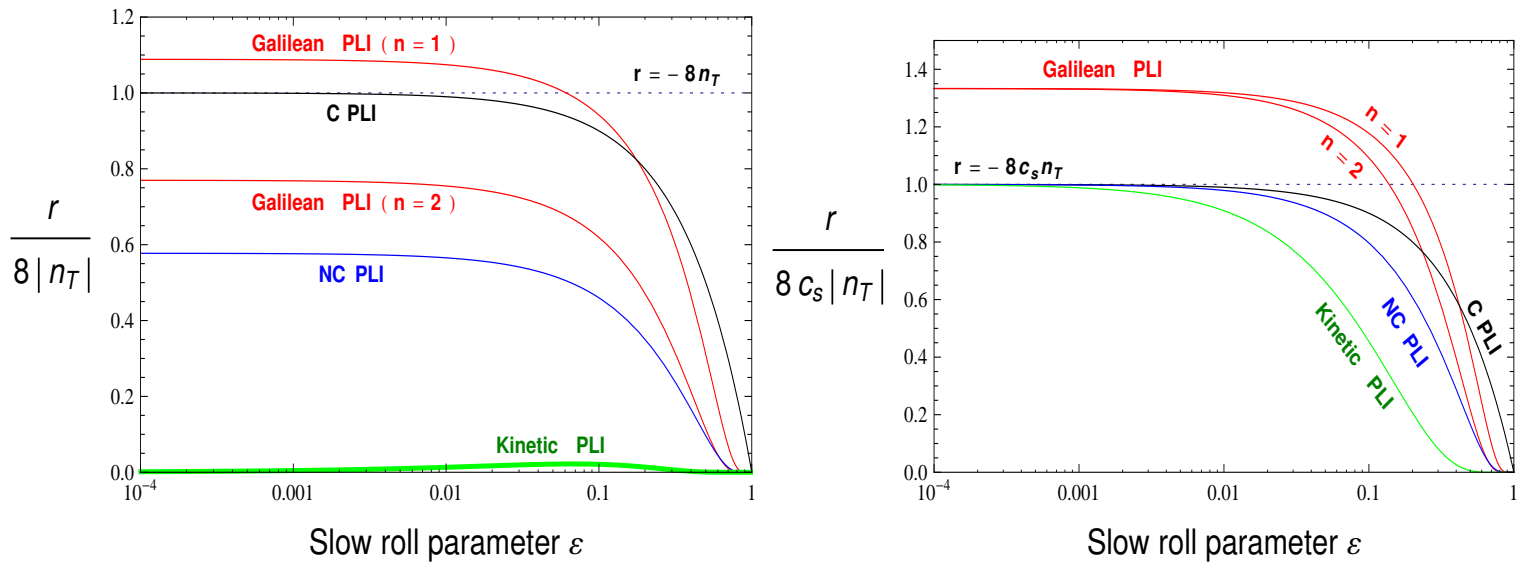

Figure 2. In the left panel, the ratio $r /\left(8\left|n_{T}\right|\right)$ is plotted as function of the slow roll parameter $\varepsilon=-\dot{H} /\left(H^{2}\right)$ for the following four power law inflation models: $(i)$ Galilean PLI: Power law inflation in Galilean model (3.1) with $n=1$ and $n=2$, $(i i)$ C PLI: Canonical scalar field driven power law inflation, (iii) NC PLI: A non-canonical scalar field model of power law inflation describe by the Lagrangian (5.1) with $\alpha=2$ and (iv) Kinetic PLI: A kinetic driven power law inflation model [14, 15] where $\mathcal{L}=f(\phi)\left(-X+X^{2}\right)$ and $f(\phi) \propto \phi^{-2}$. In the right panel, the ratio $r /\left(8 c_{s}\left|n_{T}\right|\right)$ is plotted for the same set of power law inflation models. Note that, in the slow roll limit, the ratio $r /\left(8 c_{s}\left|n_{T}\right|\right)$ approaches unity for canonical, kinetic and non-canonical models whereas, for the Galilean model of power law inflation, this ratio approaches $4 / 3$ irrespective of the value of $n$ in the Lagrangian (3.1).

where the expression for $c_{s}^{2}$ and $\lambda$ for the Galilean power law inflation model (3.1) are given by Eqs. (4.19) and (4.20), respectively. Note that the above consistency relation is an exact result since no slow roll approximation is imposed.

In Fig. 2, the ratios $r /\left(8\left|n_{T}\right|\right)$ and $r /\left(8 c_{s}\left|n_{T}\right|\right)$ are plotted as a function of slow roll parameter $\varepsilon \equiv-\dot{H} / H^{2}$ for different models of power law inflation. In this figure, relation (4.31) is used for the Galilean power law model while for the power law scenario in k-inflation [15] and in the non-canonical scalar field model [32], the same relation (4.31) is used but with $\lambda=1$. This is justified from the analysis of Ref. [14]. For the power law inflation driven by a canonical scalar field $r=16 / q[48]$ which leads to the following exact consistency relation $r=-8 n_{T}(q-1) / q$.

In the slow roll limit which corresponds to $q \gg 1$, the consistency relation (4.31) reduces to

$$
r \simeq-\frac{8 c_{s} n_{T}}{\lambda^{2}}
$$

In comparison, for the case of k-inflation, one gets $r=-8 c_{s} n_{T}$. Therefore, as mentioned earlier, it is the $\lambda$ parameter defined in Eq. (4.17) which alters the consistency relation in G-inflation. Note that, although, the consistency relation (4.32) was derived for the power law inflation, it is approximately valid, in the slow roll limit, for a generic G-inflation model with Lagrangian (2.2). For the power law inflation driven by a Galilean field, the slow roll regime of the consistency relation (4.32) can be re-expressed as

$$
r \simeq-8 n_{T}\left(\frac{4}{9}\right) \sqrt{\frac{6}{n}} .
$$


When $n=1$, the above relation becomes $r \simeq-(32 / 9) \sqrt{6} n_{T}$ consistent with those derived in Ref. [30]. For any value of $n$, Eq. (4.33) can also be expressed as $r \simeq(64 / 9)(\sqrt{6 / n}) \varepsilon$, where $\varepsilon$ is the slow roll parameter, which for the power law solution $a(t) \propto t^{q}$ turns out to be $\varepsilon=q^{-1}$. Although, we considered the Galilean power law inflation model (3.1) for which $G(X, \phi) \propto X^{n}$, the expression $r \simeq(64 / 9)(\sqrt{6 / n}) \varepsilon$ is also valid in case of Higgs G-inflation model where $G(X, \phi) \propto \phi^{2 n+1} X^{m}$ as described in Ref. [49]. However, recall that the expression $r \simeq(64 / 9)(\sqrt{6 / n})$ is slow roll limit of the exact relation (4.31).

When $n=1$, Eq. (4.33) implies that $r>-8 n_{T}$ even though the speed of sound is subluminal $\left(c_{s}^{2}=2 / 3\right)$. This does not arise in the case of canonical and non-canonical scalar field models of inflation for which $r \leq-8 n_{T}$ when $c_{s}^{2} \leq 1$ [14]. This leads us to ask the following important question: What is the physical reason behind the violation of the standard consistency relation in G-inflation models ? To go about answering this question let us re-write the scalar power spectrum (4.25) as

$$
\mathcal{P}_{S}(k)=\lambda^{2}{ }^{(n c)} \mathcal{P}_{S}(k),
$$

where ${ }^{(n c)} \mathcal{P}_{S}(k)$ is the scalar power spectrum that one gets in an equivalent non-canonical model of inflation which leads to the same background evolution, viz. $a(t) \propto t^{q}$ and for which the value of $c_{s}^{2}$ is the same as given in Eq.(4.19). Note that the expression for the tensor power spectrum (4.26) remains unchanged for an equivalent non-canonical scalar model, as tensor perturbations do not directly couple with scalar perturbations. In the slow roll limit the expression (4.20) implies that $\lambda \simeq \sqrt{3 / 4}$. therefore in the slow roll limit Eq. (4.34) implies that $\mathcal{P}_{S}(k)<{ }^{(n c)} \mathcal{P}_{S}(k)$. This means that the scalar power spectrum in G-inflation models is suppressed by a factor $\lambda^{2}$ as compared to the same in an equivalent k-inflation scenario and consequently this enhances the tensor-to-scalar ratio. It is because of this enhancement of tensor-to-scalar ratio, one can get $r>-8 n_{T}$ in G-inflation models even when $c_{s}^{2} \leq 1$.

Note that for integer value of $n$, it follows from Eq. (4.33) that $r>-8 n_{T}$ only when $n=1$. For $n \geq 2$ one gets back the standard consistency relation $r<-8 n_{T}$ as in the case of inflation driven by a canonical or non-canonical scalar field.

From Eqs. (4.19), (4.20) and (4.31), it follows that, if we restrict $c_{s}^{2} \leq 1$, then

$$
\frac{r}{8\left|n_{T}\right|} \leq \frac{4}{3}
$$

Therefore, the upper bound on the ratio $r /\left(8\left|n_{T}\right|\right)$ for the Galilean power law model (3.1) is 1.333 , when the speed of sound for the scalar field perturbations is restricted to be subluminal. This is the main result of this paper. In comparison, for all canonical and non-canonical scalar field models of inflation, the upper bound on the ratio $r /\left(8\left|n_{T}\right|\right)$ is unity when $c_{s}^{2} \leq 1$.

Although the expression (4.35) was obtained for a restricted class of G-inflation model (3.1) driving power law inflation, it may be worthwhile to investigate whether the inequality $r /\left(8\left|n_{T}\right|\right) \leq 4 / 3$ holds for a generic G-inflation model with the Lagrangian (2.2) but for which $c_{s}^{2} \leq 1$.

\subsection{Observational viability of Galilean power law inflation}

Let us now confront the Galilean PLI model with the recent constraints from the CMB observation from Planck mission which indicate $n_{S}=0.9603 \pm 0.0073$ and $r<0.11$ at $95 \%$ 


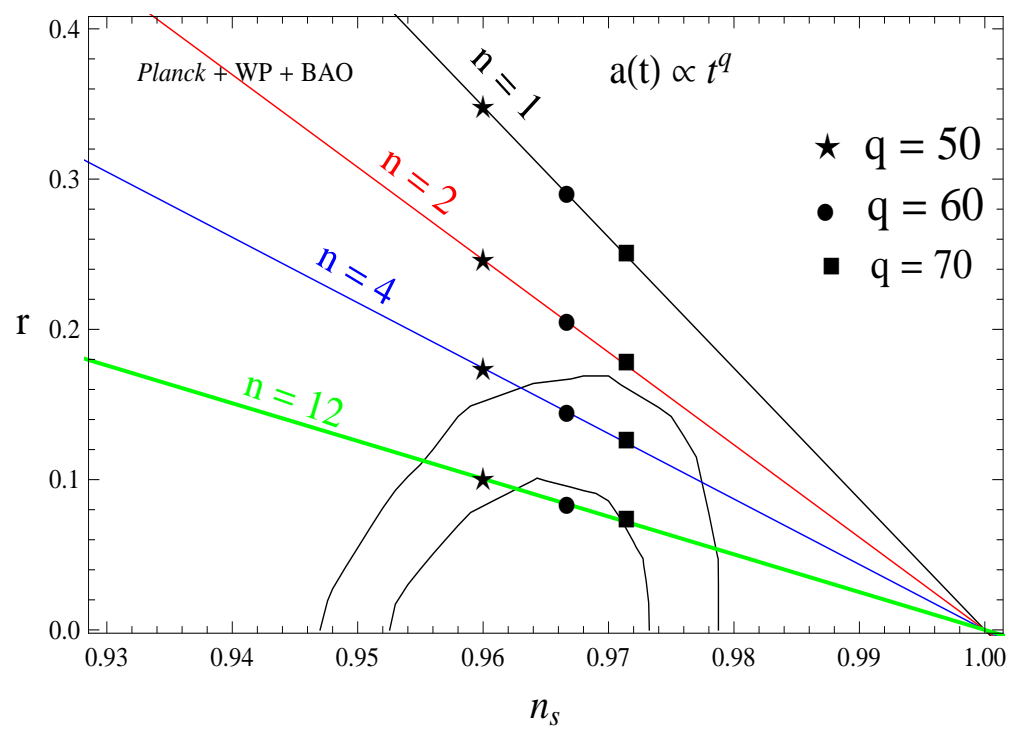

Figure 3. The constraints from the CMB observations on the power law inflation driven by a Galilean field with the Lagrangian (3.1) and with an inverse power law potential (3.10). For different values of the parameter $n$ in (3.1), the scalar spectral index $n_{S}$ and the tensor-to-scalar ratio $r$ are shown for $q=50,60$ and 70 in the power law solution $a(t) \propto t^{q}$. The inner and outer contours represents the $1 \sigma$ and $2 \sigma$ confidence limits obtained using the CMB data from Planck along with the large angle polarization data from WMAP in combination with the Baryon acoustic oscillations data $($ Planck $+\mathrm{WP}+\mathrm{BAO})[4]$.

CL [4]. In fact, at $95 \%$ CL, Planck data allows $n_{S}$ in the range [0.945-0.98]. For $n_{S}$ to lie in that range, it follows from Eq. (4.29) that $q$ in the power law solution $a(t) \propto t^{q}$ must be within the range $\sim[38-101]$. Consequently, one finds from Eq. (4.33) that $0.17 \lesssim r \lesssim 0.46$ for $n=1$, well above the bound set by the Planck results which indicate $r<0.11$ at $95 \%$ CL [4]. For example, when $n=1$ and $q=50$, Eq. (4.29) gives $n_{S} \simeq 0.96$ consistent with Planck, however, Eq. (4.33) gives a larger value of $r \simeq 0.35$. Recall that, it is for $n=1$ the Galilean power law inflation model (3.1) violates the standard consistency relation $r \leq-8 n_{T}$.

For $n=2$ and $q=100$, Eqs. (4.29) and (4.33) gives $n_{S} \simeq 0.98$ and $r \simeq 0.12$ marginally outside the Planck bounds. Since $r \propto(n)^{-1 / 2}$, larger values of $n$ lowers $r$ for a given value of $q$. For example, when $n=8$ and $q=60$, one gets $n_{S} \simeq 0.966$ and $r \simeq 0.1$ well within the Planck results which indicate $r<0.11$ at 95\% CL [4]. Therefore, for $n>2$, the Galilean power law inflation model (3.1) gives observationally consistent value for $n_{S}$ and $r$ (see Fig. 3). However, recall that for $n>2$, the model leads to $r<-8 n_{T}$.

Hence, although the Galilean power law inflation model can lead to $r>-8 n_{T}$, in the domain of the model parameter space which gives observationally viable results, the model respects the standard consistency relation $r \leq-8 n_{T}$. 


\section{Comparing Galilean and non-canonical scalar inflation}

\subsection{In the case of power inflation}

Since for an observationally viable Galilean power law inflation model $r<-8 n_{T}$, it may be possible to map this model to an equivalent scenario in a non-canonical scalar field settings ${ }^{4}$ which necessarily gives $r<-8 n_{T}$ when $c_{s}^{2}<1$. It is not possible to do so with the standard power law inflation driven by canonical scalar field with an exponential potential since for canonical inflation $r=-8 n_{T}$ and moreover the value of tensor-to-scalar ratio in such a model turns out to be larger than the bound set by the Planck results [4].

In the Galilean power law model (3.1), the speed of sound as given in Eq. (4.19) approaches a constant value in the slow roll limit and it is independent of the value of $q$ in the power law solution $a(t) \propto t^{q}$. In contrast, in the kinetic driven power law inflation model $[14,15]$ with $\mathcal{L}=f(\phi)\left(-X+X^{2}\right)$, where $f(\phi) \propto \phi^{-2}$, the speed of sound $c_{s}$ depends on the value of $q$ and in the slow roll limit one finds that $c_{s}^{2} \simeq 1 /(12 q)$. Mapping the Galilean power law model (3.1) onto the kinetic driven model depends on the value of $q$ in the power law solution $a(t) \propto t^{q}$. We therefore consider the following non-canonical model of inflation $[16,17]$

$$
\mathcal{L}(X, \phi)=X\left(\frac{X}{M^{4}}\right)^{\alpha-1}-V(\phi),
$$

where the parameter $\alpha$ is dimensionless while $M$ has mass dimension of one. The above Lagrangian can be viewed as a generalization of the standard canonical scalar field Lagrangian which corresponds to setting $\alpha=1$ in Eq. (5.1). Note that the structure of the restricted class of Galilean inflation model (3.1) is similar the above Lagrangian and astonishingly in this model also an inverse power law potential of the form [32]

$$
V(\phi)=\frac{V_{0}}{\left(\phi / M_{p}\right)^{s}} ; \quad \text { where } \quad s=\frac{2 \alpha}{\alpha-1},
$$

can drive power law inflation. The speed of sound in the model (5.1) turns out to be constant and is given by

$$
c_{S}=\frac{1}{\sqrt{2 \alpha-1}} .
$$

Since $c_{S}$ is also constant and independent of $q$ in the slow roll limit in Galilean power law inflation model, mapping between the two models is possible.

In the non-canonical power law inflation model (5.1), the spectral indices $n_{S}$ and $n_{T}$ are exactly the same as those in the Galilean model given in Eq. (4.29). However, the tensor-to-scalar ratio is different and is given by

$$
r \simeq \frac{16}{q \sqrt{2 \alpha-1}}
$$

The power law inflation model (5.1) gives observationally viable values for $n_{S}$ and $r$ for $\alpha \geq 2$ as per the recent Planck results [32]. For a given background evolution $a(t) \propto t^{q}$, the two

\footnotetext{
${ }^{4}$ It is important to note that G-inflation models $(2.2)$ with $G_{X} \neq 0$ and k-inflation models are fundamentally different scenarios. By the statement: "G-inflation power law model can be mapped to an equivalent scenario in a k-inflation model", all that we mean is that the two models leads to same set of CMBR observables $n_{S}$, $n_{T}$ and $r$.
} 
power law inflation models (3.1) and (5.1) gives the same set of value for the observables $\left\{n_{S}, n_{T}, r\right\}$, in the slow roll limit, if

$$
n=\left(\frac{32}{27}\right)(2 \alpha-1) .
$$

In other words, the two power law inflation models are observationally indistinguishable in the slow roll limit as far as the observational parameters $\left\{n_{S}, n_{T}, r\right\}$ are concerned, if the above relation is satisfied. However, it is possible that the non-gaussianity parameter $f_{\mathrm{NL}}[52]$ can act as a discriminator between the two models [55].

Although the two power law inflation models (3.1) and (5.1) give the same values for $\left\{n_{S}, n_{T}, r\right\}$, it is important to note that the speed of sound is different in both the models. In fact, for the equivalent non-canonical power law inflation model (5.1), the speed of sound turns out to be $c_{s} \times \lambda^{-2}$, where $c_{s}$ and $\lambda$ for the Galilean power law inflation model (3.1) are given in Eqs. (4.19) and (4.20), respectively. It is important to note that this is generically true in the slow roll limit for any Galilean inflation model based on the Lagrangian (2.2), which means that a Galilean model (2.2) characterized by the parameters $c_{s}$ and $\lambda$, defined in Eqs. (4.4) and (4.17), respectively, can be mapped to an equivalent k-inflation model whose speed of sound is $c_{s} \times \lambda^{-2}$. In such a scenario, the non-canonical scalar field model gives the same value for the observables $\left\{n_{S}, n_{T}, r\right\}$ as in the case of Galilean inflation. The proof the above statement is given below.

\subsection{Generic slow roll G-inflation model}

Let us consider a generic Galilean model of inflation described by the Lagrangian (2.2). From the Mukhanov-Sasaki equation (4.8), it is clear that at scales much larger than the sound horizon (ie. at $a H>>c_{s} k$ ), the mode function $u_{k} \simeq A_{k} z$, where $A_{k}$ is the $k$ dependent constant of integration. However, at scales much below the sound horizon (ie. at $a H<<c_{s} k$ ), the Bunch-Davis initial condition implies that $u_{k}=\left(2 c_{s} k\right)^{-1 / 2} \exp \left[-i k c_{s} \eta\right]$. The constant $A_{k}$ can, therefore, be fixed by equating the two regimes of solution for $u_{k}$ at the sound horizon $a H=c_{s} k$. Consequently one finds that at scales much above the sound horizon

$$
\left|\frac{u_{k}}{z}\right|^{2}=\left(\frac{1}{2 k c_{s} z^{2}}\right)_{a H=c_{s} k},
$$

where the quantity on the right hand side must be evaluated when the mode leaves the sound horizon. Substituting the above solution for $u_{k}$ in Eq. (4.10) gives

$$
\mathcal{P}_{S}(k)=\left(\frac{\lambda^{2} H^{4}}{4 \pi^{2} c_{s}\left(\rho_{\phi}+p_{\phi}\right)}\right)_{a H=c_{s} k},
$$

where $c_{s}$ and $\lambda$ are defined in Eqs. (4.4) and (4.17), respectively.

Similarly for tensor perturbations

$$
\mathcal{P}_{T}(k)=\left(\frac{2 H^{2}}{\pi^{2} M_{p}^{2}}\right)_{a H=k} .
$$

Note that unlike the case of scalar power spectrum (5.7) which is evaluated at the sound horizon $a H=c_{s} k$, the above expression for tensor power spectrum must be evaluated when the modes leaves the horizon $a H=k$. 
During slow roll inflation, $H \simeq$ constant. In addition if the variation of $c_{s}$ in the time scale of an e-fold of expansion is small enough, it is reasonable to approximate

$$
\mathrm{d} \ln k \simeq \mathrm{d} \ln a,
$$

both at the sound horizon $a H=c_{s} k$ and at the horizon $a H=k$. Consequently one finds from Eqs. (5.7) and (5.8) that

$$
\begin{aligned}
n_{S}-1 & \simeq-4 \varepsilon+2 \delta-\sigma_{s}+2 \sigma_{\lambda}, \\
n_{T} & \simeq-2 \varepsilon,
\end{aligned}
$$

where $\varepsilon, \delta, \sigma_{s}$ and $\sigma_{\lambda}$ are the first order slow roll parameters defined as

$$
\begin{aligned}
\varepsilon & \equiv-\frac{\dot{H}}{H^{2}}, \\
\delta & \equiv \varepsilon-\frac{\dot{\varepsilon}}{2 H \varepsilon}, \\
\sigma_{s} & \equiv \frac{\dot{c_{s}}}{H c_{s}}, \\
\sigma_{\lambda} & \equiv \frac{\dot{\lambda}}{H \lambda} .
\end{aligned}
$$

These slow roll parameters are generic to any scalar field models of inflation based on the Lagrangian (2.2). Depending on the form of the functions $K(X, \phi)$ and $G(X, \phi)$, it is possible to define model specific slow roll parameters, see for instance $[19,30]$.

Although the two power spectrums (5.7) and (5.8) are evaluated at two different moments in time, with the scalar power spectrum evaluated when the mode crosses the sound horizon while the tensor power spectrum evaluated when the mode leaves the horizon, one approximately gets the same results in the slow roll limit, even if both are evaluated at the horizon crossing $a H=k$. Therefore, it follows from Eqs. (5.7) and (5.8) that

$$
r=\frac{16 c_{s} \varepsilon}{\lambda^{2}} .
$$

Note that the expression for $n_{S}, n_{T}$ and $r$ as described in Eqs. (5.10), (5.11) and (5.16), respectively, are valid in the slow roll limit of G-inflation model (2.2). In the case of further generalization of G-inflation scenario which contains K-inflation models as its subclass, a detailed description of the primordial power spectra and their consequent observables $n_{S}, n_{T}$ and $r$ can be found in Ref. [53].

Let us now compare these values for $n_{S}, n_{T}$ and $r$ for non-canonical scalar field models of inflation. For consistency in notation, let the Lagrangian of the non-canonical scalar field be represented as

$$
\mathcal{L}=\widetilde{K}(X, \phi)
$$

In this model, the squared speed of sound turns out to be ${ }^{5}$

$$
{\widetilde{c_{s}}}^{2} \equiv \frac{\widetilde{K}_{X}}{\widetilde{K}_{X}+2 X \widetilde{K}_{X X}}
$$

\footnotetext{
${ }^{5}$ The notation $\widetilde{c_{s}}$ is used here to describe the speed of sound in k-inflation just to differentiate it with $c_{s}$ which represents the speed of sound in G-inflation.
} 
In a generic k-inflation model (5.17), it turns out that in the slow roll limit [14]

$$
\begin{aligned}
n_{S}-1 & \simeq-4 \varepsilon+2 \delta-\widetilde{\sigma}_{s}, \\
n_{T} & \simeq-2 \varepsilon \\
r & \simeq 16 \widetilde{c}_{s} \varepsilon,
\end{aligned}
$$

where $\varepsilon$ and $\delta$ are defined in Eqs. (5.12) and (5.13), respectively, and $\widetilde{\sigma}_{s} \equiv \dot{\vec{c}}_{s} /\left(\widetilde{c}_{s} H\right)$ is the same as those defined in Eq. (5.14), the only difference here is that $c_{s}$ in Eq. (5.14) is replaced by $\widetilde{c}_{s}$.

Let the k-inflation model (5.17) be such that it lead to the same background evolution for $a(t)$ as those in the G-inflation model (2.2). In addition, if its speed of sound is such that

$$
\widetilde{c_{s}}=\frac{c_{s}}{\lambda^{2}}
$$

where $c_{s}$ and $\lambda$ are defined in Eqs. (4.4) and (4.17), respectively, it follows from equations (5.10), (5.11), (5.16) and (5.19) to (5.21) that the observables $\left\{n_{S}, n_{S}, r\right\}$ are the same for both the models (2.2) and (5.17).

Hence, in the slow roll regime of any generic G-inflation model (2.2) one can associate a k-inflation model (5.17) whose speed of sound satisfy the relation (5.22). The two models will then be observationally indistinguishable as far as the basic set of observables $\left\{n_{S}, n_{S}, r\right\}$ are concerned.

In the case of k-inflation models, the non-gaussianity parameter in the equilateral limit is inversely proportional to the square of the speed of sound $c_{s}^{2}$ whereas the tensor-to-scalar ratio $r$ is proportional to $c_{s}$ [54]. Therefore, for a given value of the slow roll parameter $\varepsilon$, k-inflation models with smaller $r$ leads to larger $f_{\mathrm{NL}}^{\text {equil }}$. However, in the case of G-inflation models, it is possible to have a large value of $f_{\mathrm{NL}}^{\text {equil }}$ even when $r$ is large [55]. Therefore, the non-gaussianity parameter $f_{\mathrm{NL}}$ along with the other observables such as $r$ can, in principle, discriminate between the G-inflation and k-inflation scenarios [56, 57]. Note that the recent

CMB observation from Planck has indicated that $f_{\mathrm{NL}}^{\text {equil }}=-42 \pm 75$ [52] whereas $r<0.11$ at $95 \%$ CL [4].

It is also important to note that in Galilean models of inflation an additional slow roll parameter $\sigma_{\lambda}$, defined in Eq. (5.15), is introduced to describe the slow roll inflation. The slow roll approximation, at the leading order, not only corresponds to the conditions $\varepsilon<<1$ and $|\delta|<<1$, but also requires the following assumptions $\left|\sigma_{s}\right|<<1$ and $\left|\sigma_{\lambda}\right|<<1$. In the scenario when the slow roll parameters and their time variations are not small enough, it may be worth pursuing whether the generalised slow roll approach for calculating the power spectrums can be extended to Galilean models of inflation [42, 43].

\section{Summary and conclusions}

In this paper we considered a restricted class of Galilean inflation models with the Lagrangian of the form (3.1) and showed that an inverse power law potential (3.10) can lead to power law inflation. An exact inflationary consistency relation was derived for this model without imposing the slow roll condition and its evolution with the slow roll parameter $\varepsilon$ is depicted in Fig. 2. From the consistency relation, it turns out that one can have both $r>-8 n_{T}$ or $r \leq-8 n_{T}$, depending on the value of the parameter $n$ in the Lagrangian (3.1), in spite of the fact that the speed of sound is subluminal in both the cases. Interestingly, as the value 
of slow roll parameter $\varepsilon$ is varied from $\varepsilon<<1$ to $\varepsilon \simeq 1$, the consistency relation in the model which leads to $r>-8 n_{T}$ evolves towards $r \leq-8 n_{T}$. This important result is depicted in Fig. 2. Therefore, the violation of the standard consistency relation $\left(r \leq-8 n_{T}\right)$ in power law G-inflation model happens only in the slow roll regime $(\varepsilon<<1)$. Hence for investigating the violation of consistency relation in a generic G-inflation model, it is highly reasonable to impose the slow roll approximation as done in Refs. [30, 49, 53].

In the slow roll regime of power law G-inflation model (3.1), when the parameter $n \geq 2$ it turns out that $r<-8 n_{T}$, but for $n=1$ the model leads to $r>-8 n_{T}$ even though $c_{s}<1$. This is contrary to what one gets in inflation models based on a canonical or non-canonical scalar field for which $c_{s} \leq 1$ necessarily implies that $r \leq-8 n_{T}$. We have identified the reason for this distinct behavior in Galilean inflation models and is attributed to the fact that in these models the scalar power spectrum is suppressed by a factor $\lambda^{2}$, where $\lambda$ is defined in Eq. (4.17), as compared to a k-inflation model which leads to the same background evolution and has the same speed of sound for scalar field perturbations. Consequently, the tensorto-scalar ratio is enhanced by a factor $\lambda^{-2}$ which alters the inflationary consistency relation which one gets in an equivalent k-inflation model. On restricting the speed of sound in the Galilean power law inflation model (3.1) to be subluminal, we find that the upper bound on the ratio $r /\left(8\left|n_{T}\right|\right)$ is 1.333 . This is the core result of this paper.

Even though it is possible to get $r>-8 n_{T}$ in the Galilean power law inflation model, the domain of the parameter $n$ which gives observationally viable value for $n_{S}$ and $r$, as per the recent Planck results, naturally lead to $r<-8 n_{T}$ which one gets in non-canonical scalar field model of inflation. Therefore, as demonstrated in Sec. 5, one can map observationally viable Galilean model (2.2) to an equivalent non-canonical model which leads to the same background evolution but whose speed of sound satisfies relation (5.22). In such a scenario, the Galilean and the non-canonical scalar field model of inflation are observationally indistinguishable as per the basic set of observables $\left\{n_{S}, n_{T}, r\right\}$.

It is important to note that any generic k-inflation model is completely described by the behaviour of the equation of state parameter $w$ and the speed of sound $c_{s}$. However, in the case of Galilean scalar field models (2.2), an additional parameter $\lambda$ defined in Eq. (4.17) is also required to describe the system completely. The reason for the appearance of this additional parameter $\lambda$ is related to the fact that Galilean scalar field models (2.2) behaves as an imperfect fluid [50] and it is well known that additional parameters besides $w$ and $c_{s}$ are required to describe imperfect fluids. For all standard single scalar field models, canonical or non-canonical, it turns out that $\lambda=1$. In the case of inflation driven by a canonical scalar field, in addition to $\lambda=1$, the speed of sound $c_{s}=1$. The non-gaussianity parameter $f_{\mathrm{NL}}^{\text {equil }}$, evaluated in the equilateral limit, can act as an observational signature of any deviation of the speed of sound from $c_{s}=1$ [52]. Similarly, it is worth exploring whether there exists any observational consequences of deviation of the parameter $\lambda$ from $\lambda=1$, which can then act as a definitive test of Galilean models of inflation.

\section{Acknowledgments}

We thank Misao Sasaki and Varun Sahni for enlightening discussions on inflationary consistency relation and also thank the support of Max Planck-India Partner Group on Gravity and Cosmology. SS is partially supported by Ramanujan Fellowship of DST, India. 


\section{A Speed of sound of scalar field perturbations}

Here, we derive the expression for the speed of sound with which the scalar field perturbations propagates in the Galilean model (2.2). The equation of motion for the Galilean field in the curved space time is given in Eq. (2.5). However, note that this equation contains the term $R_{\mu \nu} \partial^{\mu} \phi \partial^{\nu} \phi$. This term can be expressed solely in terms of the field $\phi$ and its derivatives since Einstein's equation implies that

$$
R_{\mu \nu}=\left(\frac{1}{M_{p}^{2}}\right)\left(T_{\mu \nu}-\frac{T}{2} g_{\mu \nu}\right)
$$

Substituting for $T_{\mu \nu}$ from Eq. (2.7) in the above equation gives

$$
R_{\mu \nu} \partial^{\mu} \phi \partial^{\nu} \phi=\left(\frac{2}{M_{p}^{2}}\right)\left[X\left(K+X K_{X}\right)-4 X^{2} G_{\phi}+X^{2} G_{X} \square \phi-2 X G_{X} \partial^{\mu} \phi \partial_{\mu} X\right] .
$$

Substituting (A.2) in Eq. (2.5) we obtain the following equation of motion

$$
B_{1} \square \phi+B_{2} \partial^{\mu} \phi \partial_{\mu} X+B_{3}(2 X)+B_{4} G_{X}+B_{5} G_{X X}+K_{\phi}=0,
$$

where

$$
\begin{aligned}
& B_{1}=K_{X}-2 G_{\phi}+2 X G_{X \phi}-\frac{2 X^{2} G_{X}^{2}}{M_{p}^{2}}, \\
& B_{2}=K_{X X}-2 G_{X \phi}+\frac{4 X^{2} G_{X}^{2}}{M_{p}^{2}}, \\
& B_{3}=K_{X \phi}-G_{\phi \phi}+\left(\frac{G_{X}}{M_{p}^{2}}\right)\left(4 X G_{\phi}-X K_{X}-K\right), \\
& B_{4}=(\square \phi)^{2}-\left(\partial^{\mu} \phi\right)_{; \nu}\left(\partial^{\nu} \phi\right)_{; \mu}, \\
& B_{5}=\left(\partial^{\mu} \phi \partial_{\mu} X\right) \square \phi-\partial^{\mu} X \partial_{\mu} X .
\end{aligned}
$$

Eq. (A.3) is valid in any curved space-time. In a spatially flat FRW line element (2.8), it is straightforward to verify that Eq. (A.3) reduces to Eq. (2.17).

Let us consider the following perturbation in the scalar field

$$
\phi(\vec{x}, t)=\phi(t)+\delta \phi(\vec{x}, t)
$$

where $\phi(t)$ is the solution of the background equation (2.17). Since scalar field perturbations propagates as sound waves only at scales much below the size of the horizon, as a reasonable approximation for the derivation of $c_{s}$, one can ignore the metric perturbations and consider the evolution of $\phi(\vec{x}, t)$ defined in Eq. (A.5) in a spatially flat FRW line element (2.8). On substituting Eq. (A.5) in Eq. (A.3) and eliminating the background equation (2.17), we obtain the following equation of motion for $\delta \phi(\vec{x}, t)$ :

$$
N_{1} \ddot{\delta \phi}-N_{2}\left(\frac{\nabla^{2} \delta \phi}{a^{2}}\right)+N_{3} \dot{\delta \phi}+N_{4} \delta \phi=0
$$


where

$$
\begin{aligned}
N_{1}= & K_{X}+2 X K_{X X}-2 G_{\phi}-2 X G_{X \phi}+6 H \dot{\phi}\left(G_{X}+X G_{X X}\right)+\frac{6 X^{2} G_{X}^{2}}{M_{p}^{2}}, \\
N_{2}= & K_{X}-2 G_{\phi}+2 X G_{X \phi}+2 \ddot{\phi}\left(G_{X}+X G_{X X}\right)+4 H \dot{\phi} G_{X}-\frac{2 X^{2} G_{X}^{2}}{M_{p}^{2}}, \\
N_{3}= & 6 H G_{X}(\ddot{\phi}+2 H \dot{\phi})+G_{X X}\left(12 H^{2} X \dot{\phi}+21 X \dot{\phi} \ddot{\phi}\right)+3 H G_{X X X} \dot{\phi}^{4} \ddot{\phi}-K_{X \phi} \dot{\phi} \\
& +3 H B_{1}+2 \dot{\phi} \ddot{\phi} B_{2}+2 \dot{\phi} B_{3}+(\ddot{\phi}+3 H \dot{\phi}) \dot{\phi} B_{1 X}+2 X \dot{\phi}^{2} B_{2 X}+2 X \dot{\phi} B_{3 X}, \\
N_{4}= & 6 H \dot{\phi} G_{X \phi}(\ddot{\phi}+H \dot{\phi})+3 H \dot{\phi}^{3} \ddot{\phi} G_{X X \phi}-K_{\phi \phi}+(\ddot{\phi}+3 H \dot{\phi}) B_{1 \phi}+2 X \dot{\phi} B_{2 \phi}+2 X B_{3 \phi} .
\end{aligned}
$$

In the above equation, $B_{1}, B_{2}$ and $B_{3}$ are defined in Eq. (A.4) and the notations such as $B_{1 X}$ denotes $\partial B_{1} / \partial X$. In the Fourier space $\nabla^{2} \delta \phi=-k^{2} \delta \phi_{k}$. Therefore, at scales much below horizon, which corresponds the large $k$ limit, Eq. (A.6) approximately becomes a sound wave equation:

$$
\ddot{\delta \phi}-c_{s}^{2}\left(\frac{\nabla^{2} \delta \phi}{a^{2}}\right) \simeq 0
$$

where $c_{s}^{2}=N_{2} / N_{1}$ and therefore given by

$$
c_{s}^{2}=\frac{K_{X}-2 G_{\phi}+2 X G_{X \phi}+2 \ddot{\phi}\left(G_{X}+X G_{X X}\right)+4 H \dot{\phi} G_{X}-2 X^{2} G_{X}^{2} / M_{p}^{2}}{K_{X}+2 X K_{X X}-2 G_{\phi}-2 X G_{X \phi}+6 H \dot{\phi}\left(G_{X}+X G_{X X}\right)+6 X^{2} G_{X}^{2} / M_{p}^{2}} .
$$

For the k-inflation models which corresponds to setting $G(X, \phi)=0$ in the Lagrangian (2.2), the above equation for $c_{s}^{2}$ reduces to Eq. (5.18). In addition, if $K(X, \phi)=X-V(\phi)$, which represents canonical scalar field models one gets back the standard result $c_{s}^{2}=1$ [58].

\section{References}

[1] A. A. Starobinsky, A new type of isotropic cosmological models without singularity, Phys. Lett. B 91 (1980) 99; A. H. Guth, Inflationary universe: A possible solution to the horizon and flatness problems, Phys. Rev. D 23 (1981) 347.

[2] A. D. Linde, A new inflationary universe scenario: A possible solution of the horizon, flatness, homogeneity, isotropy and primordial monopole problems, Phys. Lett. B 108 (1982) 389; A. Albrecht and P. J. Steinhardt, Cosmology for Grand Unified Theories with Radiatively Induced Symmetry Breaking, Phys. Rev. Lett. 48 (1982) 1220; A. D. Linde, Phys. Lett. B, Chaotic inflation 129 (1983) 177.

[3] V. F. Mukhanov and G. V. Chibisov, Quantum fluctuations and a nonsingular universe JETP Lett. 33 (1981) 532; S. W. Hawking, The development of irregularities in a single bubble inflationary universe, Phys. Lett. B 115 (1982) 295; A. A. Starobinsky, Phys. Lett. B Dynamics of phase transition in the new inflationary universe scenario and generation of perturbations, 117 (1982) 175; A. H. Guth and S.-Y. Pi, Fluctuations in the New Inflationary Universe, Phys. Rev. Lett. 49 (1982) 1110.

[4] P. A. R. Ade, et. al., Planck 2013 results. XXII. Constraints on inflation, arXiv:1303.5082.

[5] P. A. R. Ade, et. al., Planck 2013 results. XVI. Cosmological parameters, arXiv:1303.5076.

[6] J. Martin, C. Ringeval and V. Vennin, Encyclopaedia Inflationaris, arXiv:1303.3787.

[7] A. Mazumdar and J. Rocher, Particle physics models of inflation and curvaton scenarios, Phys. Rept. 497, 85 (2011) [arXiv: 1001.0993]. 
[8] A. Riotto, Inflation and the theory of cosmological perturbations, arXiv: hep-ph/0210162

[9] J. Martin, Inflationary cosmological perturbations of quantum-mechanical origin, Lect. Notes Phys. 669, 199 (2005) arXiv: hep-th/0406011

[10] B. A. Bassett, S. Tsujikawa and D. Wands, Inflation dynamics and reheating, Rev. Mod. Phys. 78, 589 (2006) [arXiv: astro-ph/0507632].

[11] W. H. Kinney, TASI Lectures on inflation, arXiv: 0902.1529.

[12] L. Sriramkumar, An introduction to inflation and cosmological perturbation theory, Curr. Sci. 97 (2009) 868 [arXiv: 0904.4584].

[13] D. Baumann, TASI Lectures on inflation, arXiv: 0907.5424.

[14] J. Garriga and V. F. Mukhanov, Perturbations in k-inflation, Phys. Lett. B 458 (1999) 219 [hep-th/9904176].

[15] C. Armendariz-Picon, T. Damour and V. Mukhanov, K-Inflation, Phys. Lett. B 458, 209 (1999) [arXiv:hep-th/9904075].

[16] S. Unnikrishnan, V. Sahni and A. Toporensky, Refining inflation using non-canonical scalars, JCAP 1208 (2012) 018 [arXiv: 1205.0786].

[17] S. Li and A. R. Liddle, Observational constraints on K-inflation models, JCAP 1210 (2012) 011 [arXiv: 1204.6214].

[18] C. Deffayet, O. Pujolas, I. Sawicki and A. Vikman, Imperfect Dark Energy from Kinetic Gravity Braiding, JCAP 1010, 026 (2010) [arXiv: 1008.0048].

[19] T. Kobayashi, M. Yamaguchi and J. Yokoyama, Inflation Driven by the Galileon Field, Phys. Rev. Lett. 105, 231302 (2010) [arXiv: 1008.0603].

[20] C. Deffayet, S. Deser, G. Esposito-Farese, Generalized Galileons: All scalar models whose curved background extensions maintain second-order field equations and stress tensors, Phys. Rev. D 80, 064015 (2009) [arXiv: 0906.1967].

[21] D. Lovelock, The Einstein tensor and its generalizations, J. Math. Phys. 12, 498 (1971).

[22] A. Nicolis, R. Rattazzi and E. Trincherini, Galileon as a local modification of gravity, Phys. Rev. D 79, 064036 (2009) [arXiv: 0811.2197].

[23] C. Deffayet, G. Esposito-Farese and A. Vikman, Covariant Galileon, Phys. Rev. D 79, 084003 (2009) [arXiv: 0901.1314].

[24] A. De-Felice and S. Tsujikawa, $f(R)$ theories, Living Rev. Rel. 13, 3 (2010) [arXiv: 1002.4928].

[25] A. A. Starobinsky, Spectrum of relict gravitational radiation and the early state of the universe, JETP Lett. 30 (1979) 682.

[26] A. A. Starobinsky, Cosmic Background Anisotropy Induced by Isotropic Flat-Spectrum Gravitational-Wave Perturbations, Soviet Astronomy Lett. 11 (1985) 133.

[27] M. Sasaki and E. D. Stewart, A General Analytic Formula for the Spectral Index of the Density Perturbations Produced during Inflation Prog. Theor. Phys. 95, 71 (1996) [astro-ph/9507001].

[28] V. Mukhanov and A. Vikman, Enhancing the tensor-to-scalar ratio in simple inflation, JCAP 0602, 004 (2006) [astro-ph/0512066].

[29] E. Babichev, V. Mukhanov and A. Vikman, $k$-Essence, superluminal propagation, causality and emergent geometry, JHEP 0802, 101 (2008) [arXiv: 0708.0561]; G. Ellis, R. Maartens and M. MacCallum, Causality and the speed of sound, Gen. Rel. Grav. 39, 1651 (2007) [arXiv:gr-qc/0703121]; C. Bonvin, C. Caprini and R. Durrer, No-go theorem for k-essence dark energy, Phys. Rev. Lett. 97, 081303 (2006) [astro-ph/0606584]; J. U. Kang, V. Vanchurin and S. Winitzki, Attractor scenarios and superluminal signals in k-essence cosmology, 
Phys. Rev. D 76, 083511 (2007) [arXiv:0706.3994].

[30] K. Kamada, T. Kobayashi, M. Yamaguchi and J. Yokoyama, Higgs G-inflation, Phys. Rev. D 83, 083515 (2011) [arXiv:1012.4238].

[31] T. Kobayashi and T. Takahashi, Large Tensor-to-Scalar Ratio in Small-Field Inflation, Phys. Rev. Lett. 110, 231101 (2013) [arXiv: 1303.0242].

[32] S. Unnikrishnan and V. Sahni, Resurrecting power law inflation in the light of Planck results, JCAP in press, [arXiv: 1305.5260].

[33] J. D. Barrow, Graduated inflationary universes, Phys. Lett. B235 (1990) 40.

[34] A. Muslimov, On the scalar field dynamics in a spatially flat Friedman universe, Class. Quant. Grav. 7 (1990) 231.

[35] J. .M. Bardeen, Gauge-invariant cosmological perturbations, Phys. Rev. D 22, 1882 (1980).

[36] H. Kodama and M. Sasaki, Cosmological Perturbation Theory, Prog. Theor. Phys. Suppl. 78, 1 (1984).

[37] V. F. Mukhanov, H. A. Feldman and R. H. Brandenberger, Theory of cosmological perturbations, Phys. Rep. 215, 203 (1992).

[38] Yi-Fu Cai, D. A. Easson and R. Brandenberger, Towards a Nonsingular Bouncing Cosmology, JCAP 08, 020 (2012) [arXiv: 1206.2382].

[39] A. Naruko and M. Sasaki, Conservation of the nonlinear curvature perturbation in generic single-field inflation, Class. Quant. Grav. 28, 072001 (2011) [arXiv: 1101.3180].

[40] M. Alishahiha, E. Silverstein and D. Tong, DBI in the Sky, Phys. Rev. D 70 (2004) 123505 [hep-th/0404084].

[41] D. A. Steer and F. Vernizzi, Tachyon inflation: tests and comparison with single scalar field inflation, Phys. Rev. D 70, 043527 (2004) [arXiv: hep-th/0310139].

[42] E. D. Stewart, Spectrum of density perturbations produced during inflation to leading order in a general slow-roll approximation, Phys. Rev. D 65, 103508 (2002) [arXiv: astro-ph/0110322].

[43] W. Hu, Generalized Slow Roll for Non-Canonical Kinetic Terms, Phys. Rev. D 84 (2011) 027303 [arXiv:1104.4500].

[44] L. F. Abbott and M. B. Wise, Constraints on generalized inflationary cosmologies, Nucl. Phys. B 244 (1984) 541.

[45] F. Lucchin and S. Matarrese, Power-law inflation, Phys. Rev. D 32 (1985) 1316.

[46] V. Sahni, Scalar field fluctuations and infrared divergent states in cosmological models with power law expansion, Class. Quant. Grav. 5 (1988) L113; Energy density of relic gravity waves from inflation, Phys. Rev. D 42 (1990) 453.

[47] S. Hotchkiss, A. Mazumdar and S. Nadathur, Observable gravitational waves from inflation with small field excursions, JCAP 02, 008 (2012) [arxiv: 1110.5389]; S. Choudhury and A. Mazumdar, An accurate bound on tensor-to-scalar ratio and the scale of inflation, arXiv: 1306.4496; S. Choudhury, A. Mazumdar and S. Pal, Low \& High scale MSSM inflation, gravitational waves and constraints from Planck JCAP 07, 041 (2013) [arxiv: 1305.6398].

[48] T. Souradeep and V. Sahni, Density Perturbations, Gravity Waves and the Cosmic Microwave Background, Mod. Phys. Lett. A7 (1992) 3541 [hep-ph/9208217].

[49] K. Kamada, T. Kobayashi, T. Kunimitsu, M. Yamaguchi and J. Yokoyama, Graceful exit from Higgs G-inflation, [arXiv: 1309.7410].

[50] O. Pujolas, I. Sawicki and A Vikman, The Imperfect Fluid behind Kinetic Gravity Braiding, JHEP 1111 (2011) 156 [arXiv: 1103.5360]. 
[51] D. A. Easson, I. Sawicki and A. Vikman, When Matter Matters, JCAP 07, 014 (2013) [arXiv: 1304.3903]

[52] P. A. R. Ade, et. al., Planck 2013 Results. XXIV. Constraints on primordial non-Gaussianity, arXiv:1303.5084.

[53] T. Kobayashi, M. Yamaguchi and J Yokoyama, Generalized G-inflation: Inflation with the most general second-order field equations, Prog. Theor. Phys. 126, 511 (2011) [arxiv: 1105.5723].

[54] X. Chen, M. Huang, S. Kachru and G. Shiu, Observational Signatures and Non-Gaussianities of General Single Field Inflation, JCAP 0701 (2007) 002 [hep-th/0605045].

[55] T. Kobayashi, M. Yamaguchi and J Yokoyama, Primordial non-Gaussianity from G-inflation, Phys. Rev. D 83, 103524 (2011) [arxiv: 1103.1740].

[56] X. Gao, T. Kobayashi, M. Shiraishi, M. Yamaguchi, J. Yokoyama and S. Yokoyama, Full bispectra from primordial scalar and tensor perturbations in the most general single-field inflation model, Prog. Theor. Exp. Phys. 053E03 (2012) [arxiv: 1207.0588].

[57] K. Kamada, T. Kobayashi, T. Takahashi, M. Yamaguchi and J. Yokoyama, Generalized Higgs inflation, Phys. Rev. D 86, 023504 (2012) [arxiv: 1203.4059].

[58] W. Hu, Structure Formation with Generalized Dark Matter, Astrophys. J 506, 485 (1998) [arXiv: astro-ph/9801234]. 